\title{
APOPTOSIS AND CELL PROLIFERATION IN CHRONIC TONSILLITIS AND OROPHARYNGEAL CARCINOMA: ROLE OF NITRIC OXIDE AND CYTOKINES
}

\author{
Tomáš Kučera* , Hana Pácováb, David Veselýb, Jaromír Astl ${ }^{\mathrm{b}}$, Jindřich Martínek ${ }^{\mathrm{a}}$
}

a Institute of Histology and Embryology and

${ }^{b}$ Department of Otorhinolaryngology and Head and Neck Surgery, $1^{\text {st }}$ Medical Faculty, Charles University, Prague, Czech Republic

e-mail: tkucer@lfl.cuni.cz

Received: September 20, 2004

Key words: Carcinogenesis/Nitric oxide synthase/Tonsillitis/Apoptosis/Cell proliferation

\section{INTRODUCTION}

Both chronic inflammation and malignant transformation are biological processes, which are regulated by various components of intra- and intercellular signaling. Although there are suspicions that inflammatory and immune responses may contribute to the development of some malignancies, the question of the relationship between inflammation and carcinogenesis is not yet resolved. The aim of this study was to determine the expression of some molecules with a potential role in both processes together with evaluation of cell proliferation and cell death. Expression of nitric oxide releasing enzyme - endothelial nitric oxide synthase (eNOS) and the expression of proliferation and apoptotic markers, $\mathrm{Ki}$ - 67 and cleaved caspase 3 respectively was detected immunohistochemically.

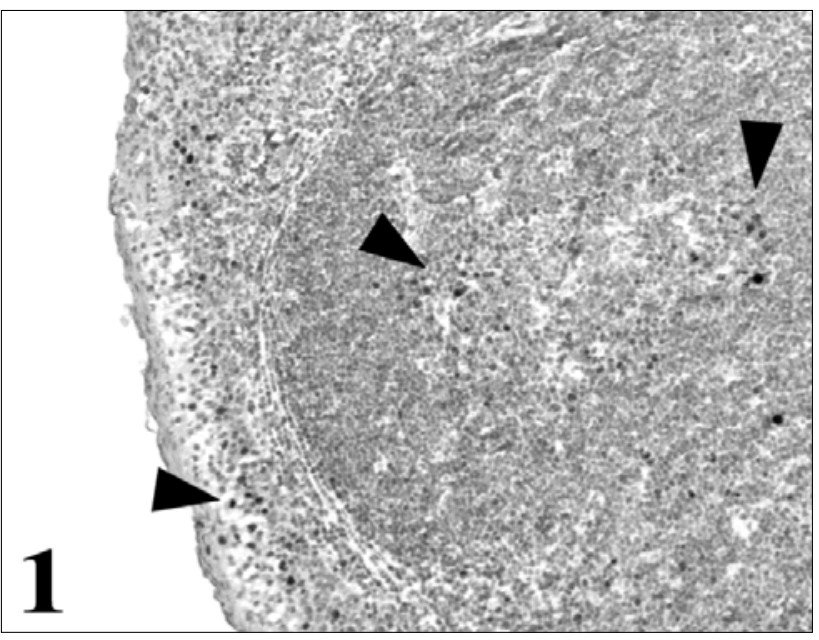

Fig. 1. Ki-67 positive nuclei (arrowheads) can be found in the surface epithelium of chronically inflamed tonsil and in the follicular germinal centers. Orig. obj. magn. $20 \mathrm{x}$

\section{MATERIAL AND METHODS}

Specimens from patients suffering from chronic tonsillitis $(n=17)$ and oropharyngeal carcinoma $(n=3)$ were fixed in $4 \%$ paraformaldehyde and embedded in paraffin for routine H-E staining and for immunohistochemistry. The three-step immunoperoxidase reaction was performed using the following antibodies: anti-eNOS rabbit polyclonal (Santa Cruz), anti Ki-67 mouse monoclonal (DAKO) and anti-cleaved caspase-3 rabbit polyclonal (Cell Signaling technology). Antibody binding was visualized by Vectastain ABC Elite kit (Vector) and diaminobenzidine as a chromogen. The sections were counterstained with hematoxylin.

\section{RESULTS AND DISCUSSION}

Examination of specimens that were taken from the tonsils of patients with chronic tonsillitis revealed frequent apoptotic cells and numerous mitotic figures in

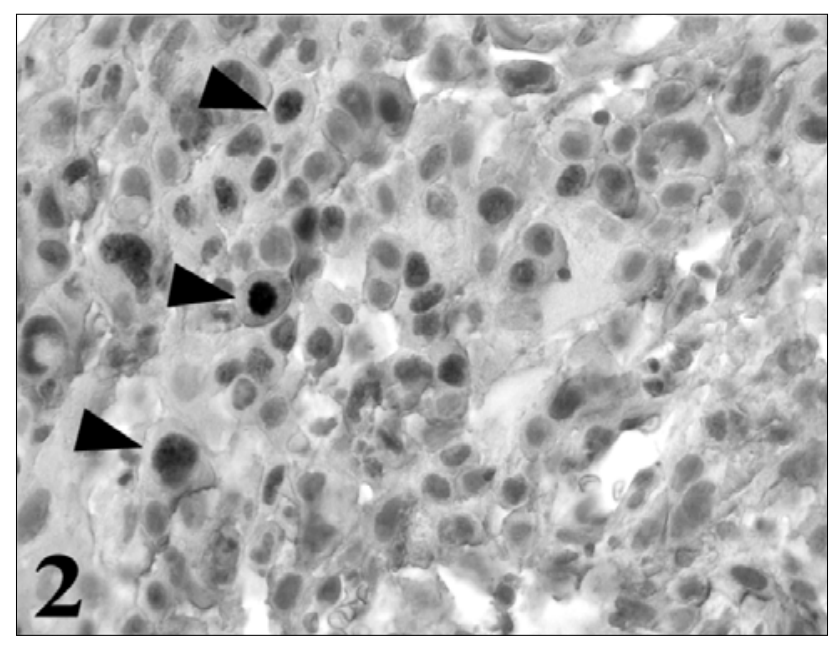

Fig. 2. Ki-67 positive cells (arrowheads) in the sample from oropharyngeal carcinoma. Orig. obj. magn. $63 \mathrm{x}$ 


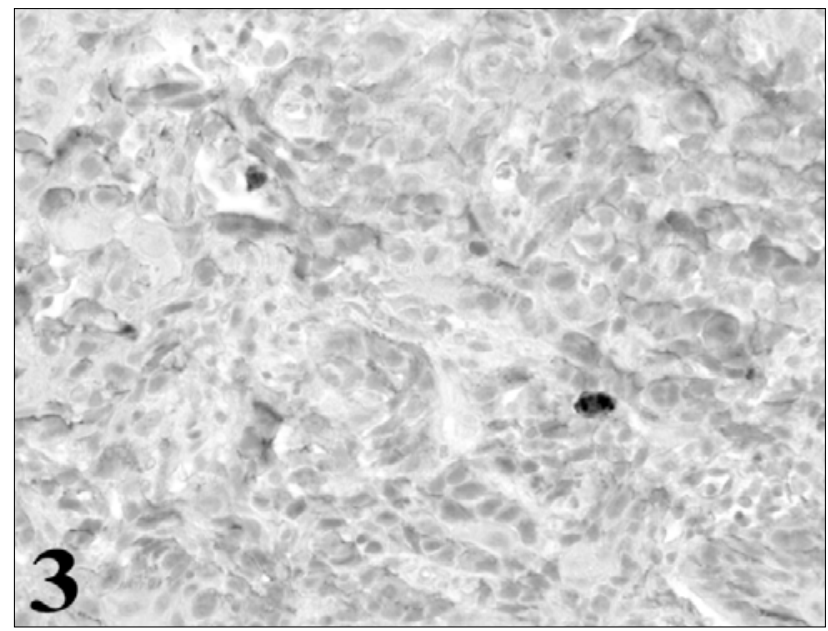

Fig. 3. Cleaved caspase-3 positivity in individual malignant cells. Orig. obj. magn. $40 \mathrm{x}$

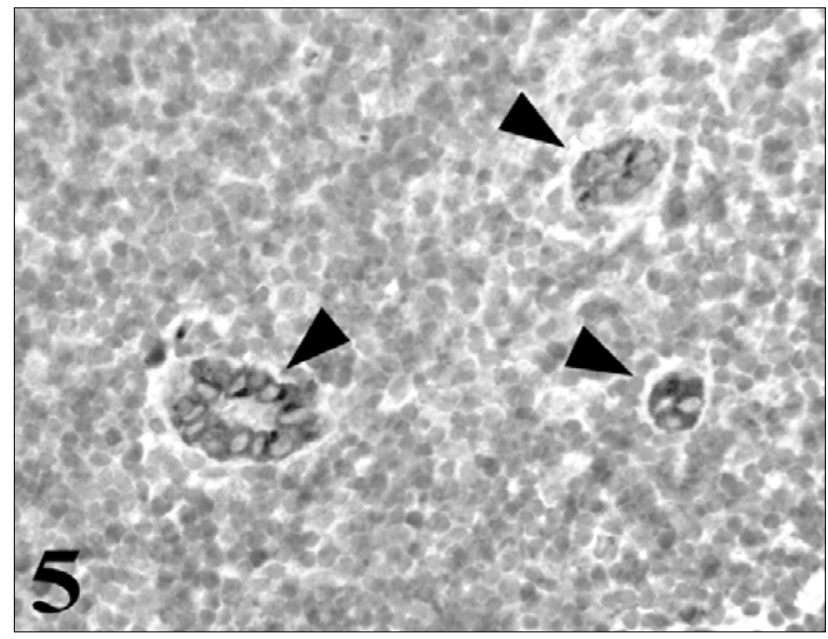

Fig. 5. Chronic tonsillitis. The immunohistochemical detection of eNOS shows strong reaction in high endothelial venules (arrowheads). Orig. obj. magn. $63 \mathrm{x}$

activated lymphoid follicles. The cell proliferation and the programmed cell death occurred less frequently in the interfollicular zone and surface epithelium of tonsils. The morphological evaluation of cell proliferation and cell death was confirmed by the immunoreactivity to the marker of proliferation, $\mathrm{Ki}-67$ and the apoptotic marker cleaved caspase-3 (Fig. 1, 4). The expression of eNOS was detected in variable amount in the vasculature of tonsillar lamina propria. The strongest immunoreaction was found in the endothelial lining of high endothelial venules (Fig. 5). In oropharyngeal carcinoma both Ki-67 and cleaved caspase-3 positive cells were scattered in the malignant tissue (Fig. 2, 3). This finding was confirmed by morphological assessment of the tissue. The positivity of eNOS was regularly observed in the highly vascularized re-

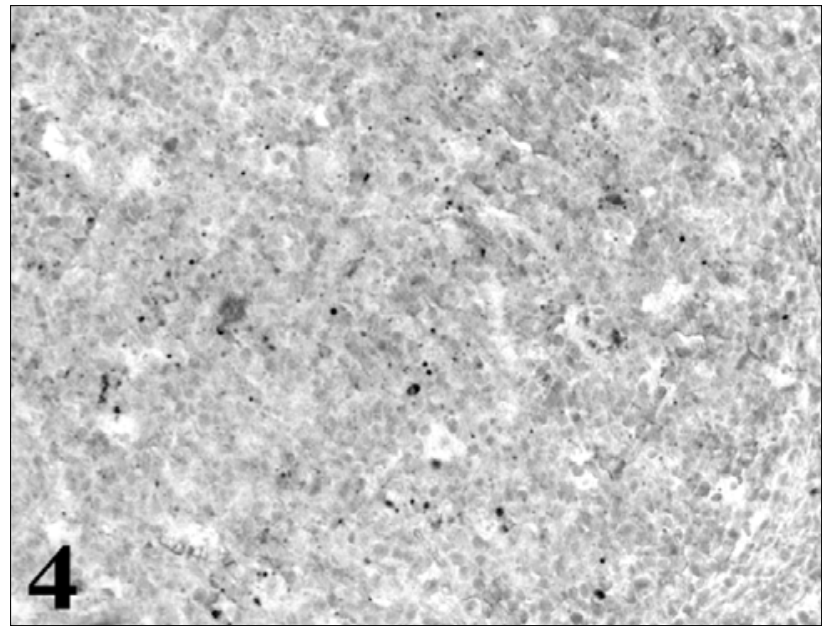

Fig. 4. Chronic tonsillitis. Apoptotic cells and apoptotic bodies in the germinal center of lymphoid follicle are positive for cleaved caspase 3 . Orig. obj. magn. $40 \mathrm{x}$

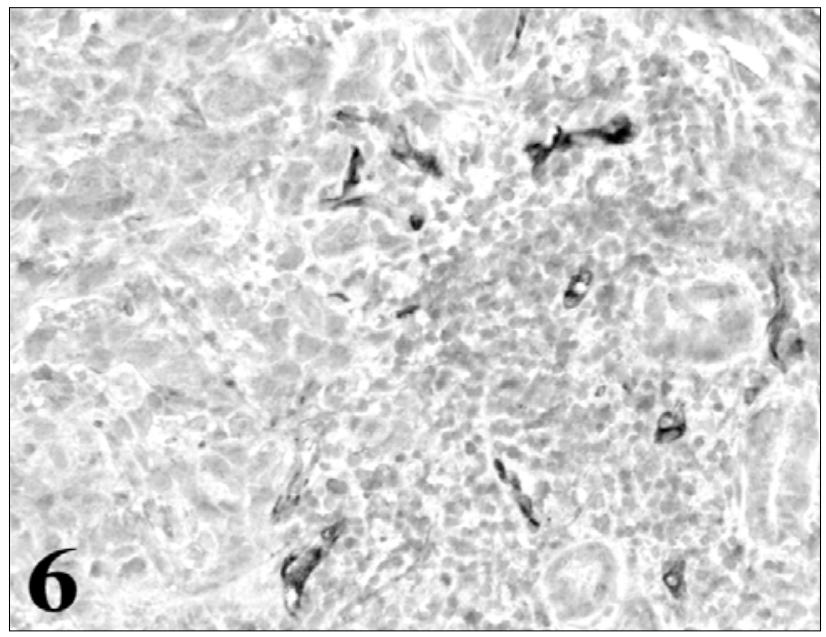

Fig. 6. Oropharyngeal carcinoma. The immunohistochemical detection of eNOS is positive in capillaries supplying the tumor. Orig. obj. magn. $40 \mathrm{x}$

gions of the tumor and it was localized in endothelial cells of capillaries supplying the tumorous mass (Fig. 6).

In both cancer and chronic inflammation in the oropharyngeal region, apoptosis is accompanied by cell proliferation. However, their distribution differs. In the case of chronically inflamed tonsil the proliferating and dying cells were prevalently maturing B-lymphocytes undergoing clonal selection. In oropharyngeal carcinoma these procceses occurred in malignant epithelial cells. Possible relations between mechanisms regulating both processes need to be resolved by further analyzes of other signaling molecules involved. One of these signaling molecules is NO that is produced by eNOS in the endothelial cells of vessels supplying the malignant tissue, where it most probably regulates blood supply. We failed 
to observe any topical association between apoptosis and proliferation on the one hand and NOS expression on the other. The expression of eNOS in high endothelial venules of the tonsillar lamina propria suggests its possible role in regulating the circulation of lymphocytes.

\section{ACKNOWLEDGEMENTS}

This work was supported by the grants GAUK 2/2004/C/ 1.LF, NC 7487-3 of IGA of the Health Ministry of the Czech Republic and the Research Project J 13/98 111100002-6 of the Ministry of Education, Youth and Sports of the Czech Republic. 